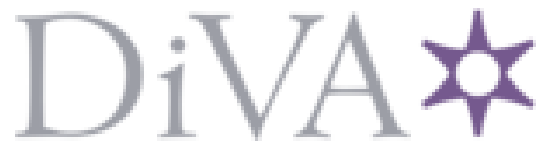

http://www.diva-portal.org

Preprint

This is the submitted version of a paper presented at 2012 IEEE International Symposium on Electrical Insulation, 1O-13 June 2012, San Juan, PR, USA.

Citation for the original published paper:

Werelius, P., Cheng, J., Ohlen, M., Robalino, D M. (2012)

Dielectric Frequency Response Measurements and Dissipation Factor Temperature

Dependence

In: (pp. 296-300).

N.B. When citing this work, cite the original published paper.

Permanent link to this version:

http://urn.kb.se/resolve?urn=urn:nbn:se:kth:diva-303020 


\section{Dielectric Frequency Response Measurements and Dissipation Factor Temperature Dependence}

\author{
Peter Werelius \\ Megger \\ Täby, Sweden \\ Peter.werelius@megger.com \\ Matz Ohlen \\ Megger \\ Täby, Sweden \\ Matz.ohlen@megger.com
}

\author{
Jialu Cheng \\ Megger \\ Täby, Sweden \\ Jialu.cheng@megger.com \\ Diego M. Robalino \\ Megger \\ Dallas, USA \\ Diego.robalino@megger.com
}

\begin{abstract}
The condition of the insulation is an essential aspect for the operational reliability of electrical power transformers, generators, cables and other high voltage equipment. Transformers with high moisture content can not without risk sustain higher loads. Bushings and cables with high moisture content at high temperature can explode due to "thermal runaway".

Typically, dissipation factor (DF) or power factor (PF) test at power frequency $50 / 60 \mathrm{~Hz}$ is carried out in the field following well known procedures. DF measured values are then normalized to $20^{\circ} \mathrm{C}$ for comparisons with guidelines and trending. However, the temperature correction factor for the normalization of the field measurement is questioned because cellulose with different moisture contents as well as oil with different conductivity will have different correction factors. It has been proved that good insulation has less temperature dependent response than the bad insulation.

DFR modeling, accurately taking the temperature effect into account, can be used to model the temperature dependence of DF over a wide temperature range based on measurements over a frequency range. Naturally, from such modeling, also temperature correction factors for correcting a measurement values obtained at one temperature, e.g. $32 \mathrm{C}$, to a reference temperature, e.g. 20C, can be calculated.

This paper will provide a background of DFR and its modeling specifically applied to model the effect of temperature and calculating temperature correction factors based on actual DFR response. The theoretical basis is backed up with several case studies of measurement on samples as well as on real objects, e.g. bushing and transformers at multiple temperatures.
\end{abstract}

Keywords- Dielectric frequency response; DFR; frequency domain spectroscopy; FDS; power factor; dissipation factor; temperature correction; temperature dependence

\section{INTRODUCTION}

With an aging power component population, today's electrical utility industry faces a tough challenge as failures, consequent repair and revenue loss may inflict major costs.
Transformers are by far, one of the most mission critical components in the electrical grid. The need for reliable diagnostic methods drives the world's leading experts to evaluate new technologies that improve reliability and optimize the use of the power network.

The condition of the insulation is an essential aspect for the operational reliability of electrical power transformers, generators, cables and other high voltage equipment. Transformers with high moisture content can not without risk sustain overloading conditions. Bushings and cables with high dissipation factor (DF) / power factor (PF) at high temperature can explode due to "thermal runaway".

On the other hand it is also very important to identify "good" units among the aging population of equipment. Adding just a few "extra" service years to the expected service life of an electrical device implies substantial cost savings for the power industry.

The widely accepted and most common insulation diagnostic test is carried out measuring capacitance and DF/PF at line frequency $(50 / 60 \mathrm{~Hz})$. This test is performed whenever there is a need for investigating insulation properties. Analysis is based on historical values (trending analysis) and comparing against factory values. Since insulation properties are temperature dependent, temperature compensation has to be used for measurements not performed at $20^{\circ} \mathrm{C}$, this is normally achieved using temperature correction table factors for certain classes of devices [1].

However, the standard table values for temperature correction are only average values and therefore subject to some error [2, 3]. The DFR of dry insulation is less temperature dependent while wet insulation is very much affected by the temperature. The insulation of the power transformers is even more complicated. It consists of mineral oil and cellulose which have different temperature characteristics. Therefore, the correction factors are usually different for different transformers depending on the design 
and individual aging.

\section{TEMPERATURE DEPENDENCE}

To be able to find the correct temperature correction factor, the temperature dependence of the insulation system must be investigated. The susceptibility of the insulation material can be expressed as a function of frequency and temperature [4]:

$$
X(\omega, T)=A(T) \cdot F\left(\frac{\omega}{\omega_{c}(T)}\right)
$$

$A(T)$ is a temperature dependent amplitude factor, $F(x)$ a spectral function and $\omega_{\mathrm{c}}(\mathrm{T})$ a characteristic frequency. $\mathrm{A}(\mathrm{T})$ is constant for cellulose. It means that the shape of the spectrum remains unchanged at different temperatures. Some insulation materials change the shape of their spectrum when temperature changes.

Most insulation materials are temperature dependent and in general, the increase/decrease of temperature makes the dielectric spectrum shift towards higher/lower frequencies. However, the shape is usually not changed. In the special case of an ideal Debye function, the complex permittivity can be written as:

$$
\varepsilon=\varepsilon_{\infty}+\frac{\Delta \varepsilon}{1+j \omega \tau \exp \left(-W_{\mathrm{a}} / \mathrm{k} T\right)}
$$

where $W_{\mathrm{a}}$ is the activation energy, $\mathrm{k}$ is the Boltzmann constant; and, $\tau$ is the relaxation time. From (2) it is clear that the permittivity is a function of $-W_{a} / \mathrm{k} T$ in logarithmic scale. The dielectric response moves to higher frequency with temperature increase, or conversely, to higher temperature as frequency increases. One can obtain the same effect as increasing the frequency or increasing the temperature. The activation energy of the Kraft paper is about 1-1.05 eV [5] while mineral oil has activation energy of $0.4-0.5 \mathrm{eV}$ [6]. Increasing temperature has the same effect on the dielectric response as decreasing frequency.

The general shape of the curve is often preserved if the data is plotted on a log-log scale. This feature allows the formation of a "Master Curve" by shifting the curves along the frequency axis until they form a continuous curve at a chosen reference temperature [5]. How to obtain the master curve is shown in Fig. 1. The dielectric frequency response of the cellulose is measured at various temperatures. Then the curves are shifted until all data points fall on the same curve: the master curve. The actual fitting of the master curve is done numerically by an algorithm that allows us to take into account both the real and imaginary part of the data. In the curve fitting process, the activation energy is calculated. An example of finding the activation energy is shown in Fig. 2.

The frequency-temperature dependence curves for three different activation energies are shown in Fig. 3. Equation (1) indicates that the increase of temperature has the same effect as the increase of frequency. To what extent they are related to each other is represented by the activation energy. The curves are examples showing that for a certain increase of temperature, the material with larger activation energy needs to be measured at higher frequency in order to obtain the same permittivity.

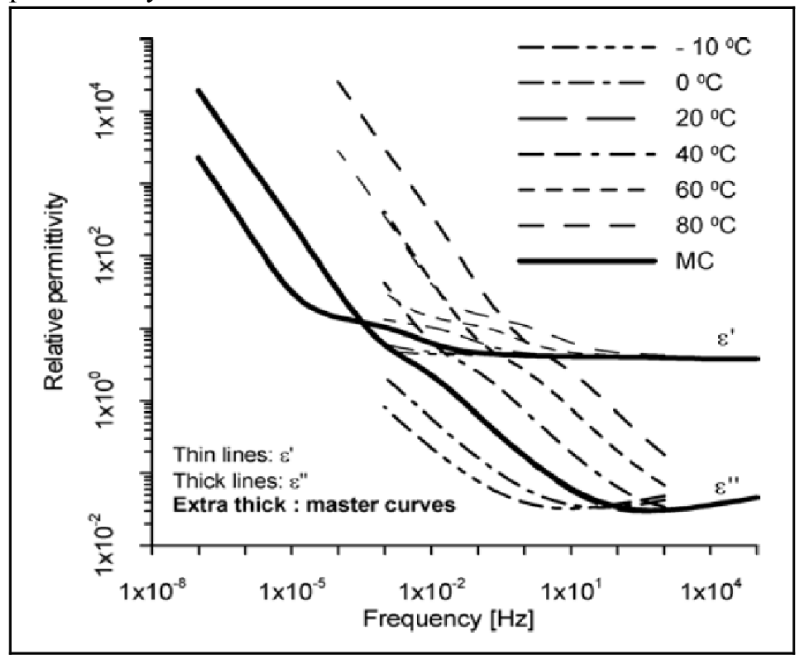

Figure 1. Example of making the master curve [5]

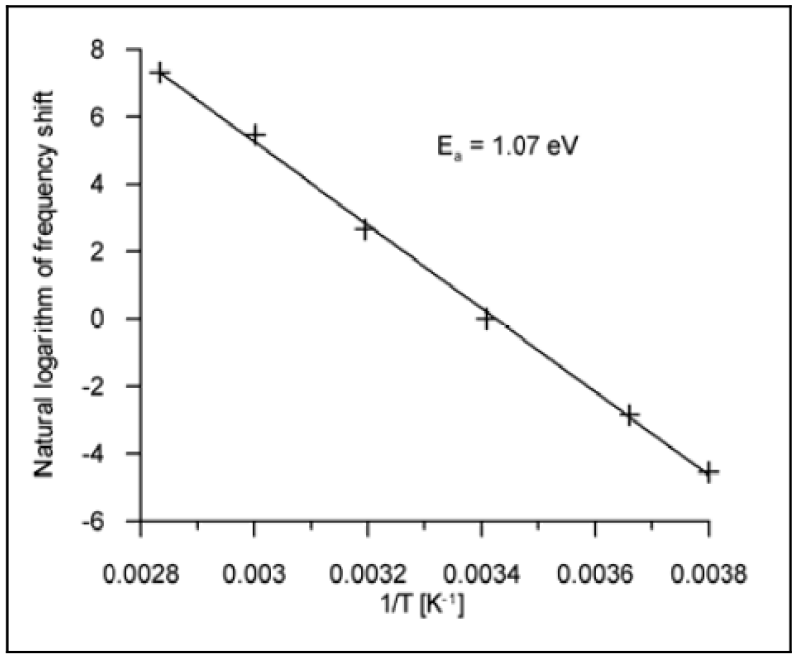

Figure 2. Example of plotting of frequency shifts to find the activation energy from the slope [5] 


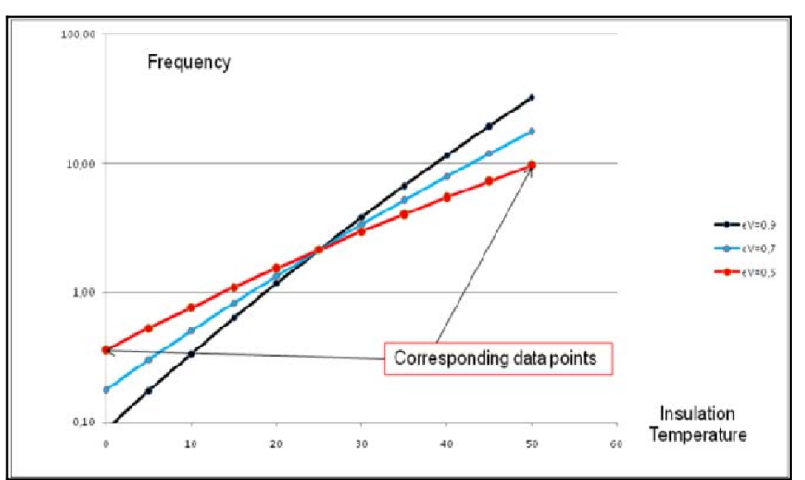

Figure 3. Relationship between power factor values at different frequencies taken at different temperatures.

\section{DIELECTRIC FREQUENCY RESPONSE MEASUREMENTS}

A field instrument for DFR measurements of transformers, bushings and cables was introduced in 1995 [7]. Since then numerous research groups have evaluated DFR technology used for field measurement. Several international projects/reports define dielectric response measurements together with insulation modeling as the preferred method for measuring moisture content of the cellulose insulation in power transformers [8], [9], and [10].

The results are normally presented as capacitance and/or dissipation factor/power factor versus frequency. DFR results obtained from a variety of transformers under different conditions are shown in Fig. 4.

Samples of Kraft paper with various moisture contents were measured at different temperatures [11]. Results for dry paper, moisture content $<0.5 \%$ are shown in Fig. 5 .

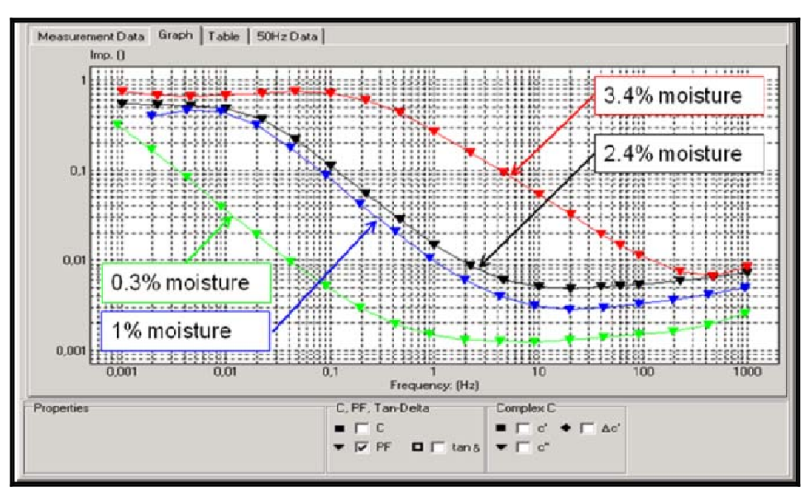

Figure 4. DFR measurement examples. our different transformers with moisture content ranging from 0.3 to $3.4 \%$

The dissipation factor at $50 \mathrm{~Hz}$ as a function of temperature for dry Kraft paper measured as a function of frequency at several temperatures is presented in Fig. 6. This analysis clearly demonstrates that the typical correction factors are not correct for an individual transformer or bushing.

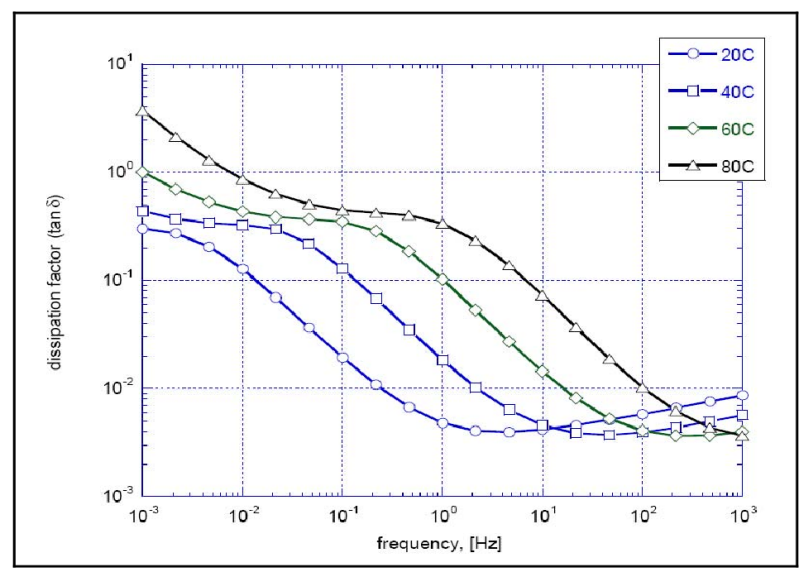

Figure 5. Dissipation factor as function of frequency for dry Kraft paper.

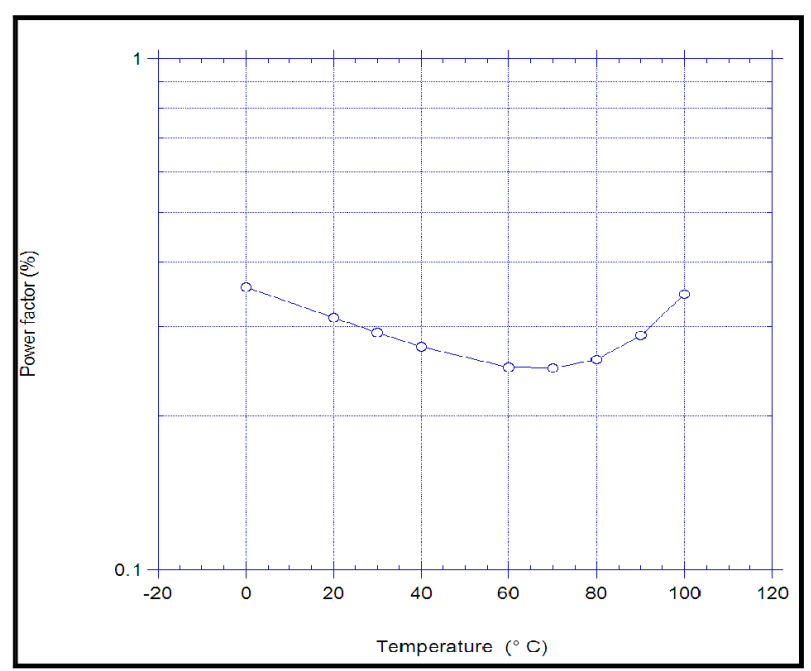

Figure 6. Dissipation factor $(50 \mathrm{~Hz})$ as function of temperature for dry Kraft paper.

The combined type insulation system of power transformers consisting of paper/pressboard and oil requires deeper investigation. In this complex insulation system, the temperature correction factors are a combination of temperature dependence of cellulose and temperature dependence of oil. Also, the amount of cellulose and oil need to be taken into account.

DFR measurement combined with mathematical modeling of the dielectric response using the so-called XY model is capable of evaluating the temperature dependence of the insulation system. The insulation structure of the power transformer is represented by the relative amount of spacers (sticks) and barriers in the cooling duct as shown in Fig. 7. Parameter " $\mathrm{X}$ " is defined as the ratio of the sum of all barriers in the duct, lumped together, and divided by the duct width. The spacer coverage "Y" is defined as the total width of all the spacers divided by the total length of the periphery of the 
duct.

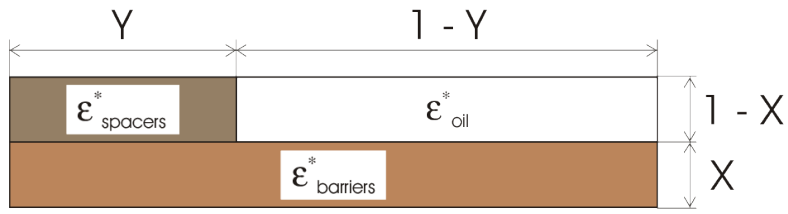

Figure 7. XY model of the transformer [6]

The permittivity of oil, $\varepsilon_{\text {oil }}^{*}$, spacers, $\varepsilon_{\text {spacers }}^{*}$ and barriers, $\mathcal{E}_{\text {barriers }}^{*}$, are complex functions of both frequency and temperature. The equivalent permittivity of the XY model is given by:

$$
\mathcal{E}(\omega, T)=\frac{Y}{\frac{1-X}{\varepsilon_{\text {spacer }}}+\frac{X}{\varepsilon_{\text {barrier }}}}+\frac{1-Y}{\frac{1-X}{\varepsilon_{\text {oil }}}+\frac{X}{\varepsilon_{\text {barrier }}}}
$$

Using DFR for moisture determination is based on the comparison of the transformers dielectric response to a modeled dielectric response. An analyzing algorithm rearranges the modeled dielectric response and delivers a new modeled curve that reflects the measured transformer. Results are presented as moisture content and oil conductivity for the transformer.

An example is shown in Fig. 8. The transformer is a 20 MVA distribution transformer. Moisture content of cellulose and oil conductivity were obtained by varying the parameters in the model curve to the measured dielectric frequency response at a certain insulation temperature.

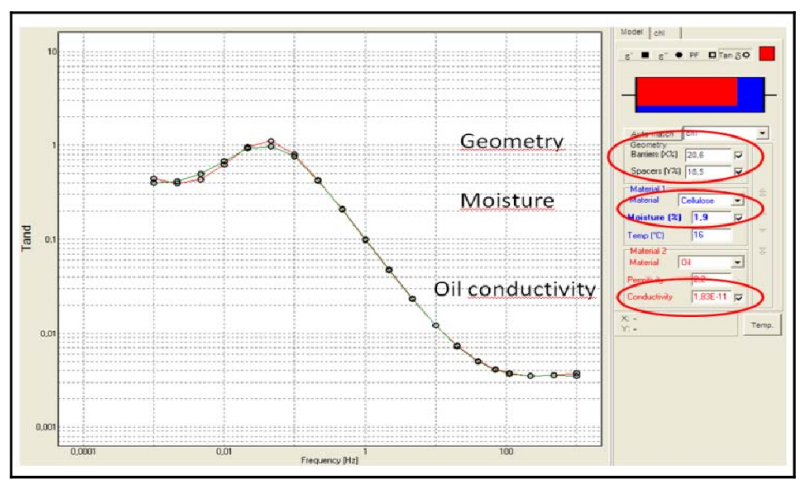

Figure 8. MODS $₫$ moisture analysis for a 20 MVA transformer

Based on the model curve, the dissipation factor as a function of temperature for that specific 20 MVA transformer is shown in Fig. 9. Figure 10 compares the dissipation factor at power frequency as function of temperature for oil, cellulose and for a combined oil and paper insulation. In this specific example, the oil temperature dependence dominates but the influence of oil and cellulose varies case by case.

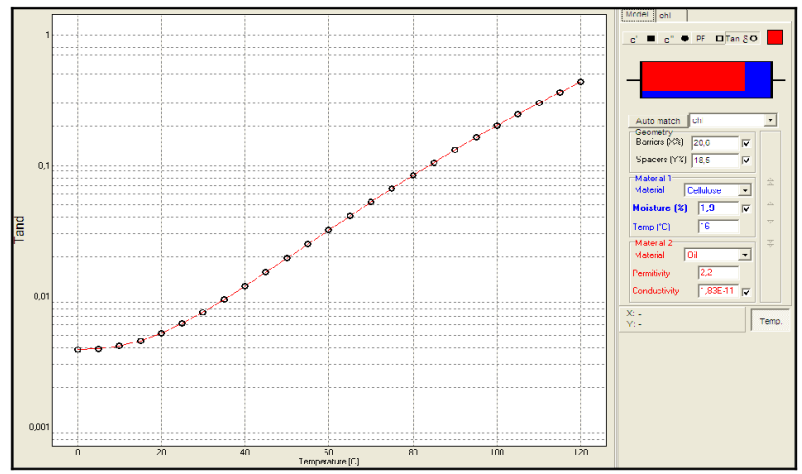

Figure 9. Dissipation factor $(50 \mathrm{~Hz})$ as function of temperature for a 20MVA distribution transformer

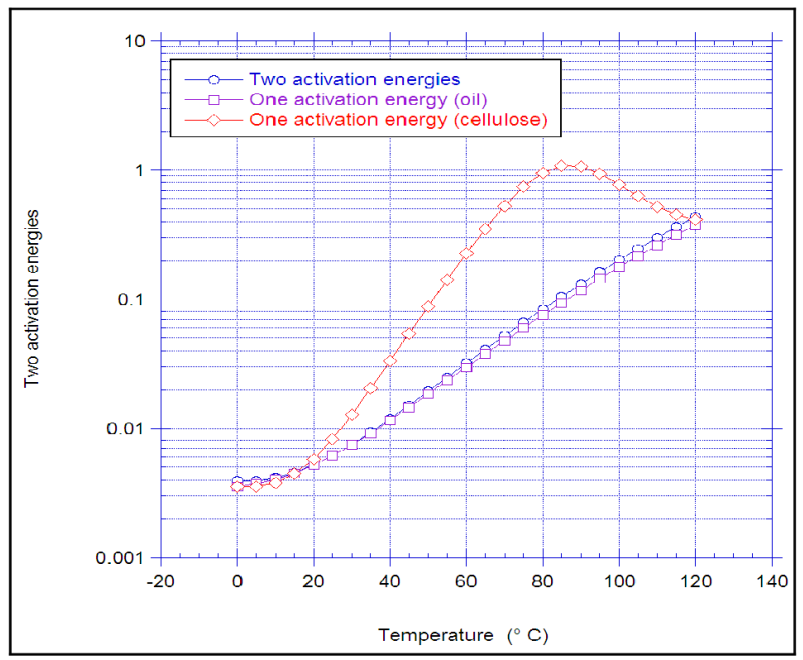

Figure 10. Relationship between dissipation factor values at different temperatures for materials with different activation energy.

Moisture analysis (moisture in paper insulation) performed on the same specimen at different temperatures may show slight deviation. Combining the modeling results and converting to temperature dependence, temperature curves can be obtained. Also, for any insulation system containing two different temperature dependent materials, the applied conversion considering activation energy of the system provides results matching very close to the actual measured dissipation factor values (tan delta) at different temperatures.

\section{SUMMARY AND CONCLUSIONS}

The dielectric frequency response in terms of capacitance and dissipation factor of an electric insulation material or an insulation system depends not only on insulation condition but also on temperature. When restricted to power frequency measurements the effect of temperature is unknown. 
Fortunately, most insulation materials have a simple relation between capacitance and dissipation factor measured as a function of temperature and as a function of frequency that can be effectively used when predicting the temperature effect on the insulation system by measuring the dielectric response of the insulation system as a function of frequency. This relation become a bit more complex, but still manageble, for an insulation system consisting of e.g. impregnated cellulose and insulating liquids as it is the case in power transformers.

The temperature-frequency model which takes into account the effect of temperature is essential because it makes the dielectric frequency response measurement an accurate tool for estimating the moisture in the cellulose of an insulation system.

The application of dielectric frequency response can also be used to estimate dissipation factors at other temperatures, e.g. a reference temperature of e.g. $20^{\circ} \mathrm{C}$ or at a higher operating temperature to evaluate the possible risk of thermal runaway.

\section{REFERENCES}

[1] IEEE Guide for Diagnostic Ficld Testing of Electric Power Apparatus; Part 1: Oil Filled Power Transformers, Regulators, and Reactors", IEEE 62-1995

[2] "Swedish Bushings Plant Sees Growth in RIP Designs", INMR Quarterly, Issue 68, 2005
[3] R.K.Tyagi, S. Victor, N.S.Sodha, "Application of Temperature Correction Factors for dissipation factor Measurements for Power Transformers - A case study", Doble Client Conference, Vadodara, India 2006

[4] A.K.Jonscher, "Dielectric Relaxiation in Solids",

[5] D. Linhjell, L. Lundgaard, U.Gäfvert, "Dielectric Response of Mineral Oil Impregnated Cellulose and the Impact of Aging", IEEE Transactions on Dielectrics and Electrical Insulation, Volume: 14 Issue:1, 2007

[6] Neimanis, R.; Arvidsson, L.; Werelius, P., "Dielectric Spectroscopy Characteristics of Aged Transformer Oils", Electrical Insulation Conference and Electrical Manufacturing \& Coil Winding Technology Conference, 2003.

[7] P. Werelius et al, "Diagnosis of Medium Voltage XLPE Cables by High Voltage Dielectric Spectroscopy", paper presented at ICSD 1998.

[8] U. Gäfvert et al, "Dielectric Spectroscopy in Time and Frequency Domain Applied to Diagnostics of Power Transformers", 6th International Conference on Properties and Applications of Dielectric Materials, June 21-26, 2000, Xi'an, China.

[9] S.M. Gubanski et al, "Dielectric Response Methods for Diagnostics of Power Transformers", Electra, No. 202, June 2002, pp 23-34,also in CIGRE Technical Brochure, No. 254, Paris 2004

[10] S.M. Gubanski et al, "Reliable Diagnostics of HV Transformer Insulation for Safety Assurance of Power Transmission System. REDIATOOL - a European Research Project", paper D1-207 CIGRE 2006

[11] R. Neimanis, T. Saha, and R. Eriksson, "Determination of moisture content in mass impregnated cable insulation using low frequency dielectricspectroscopy," IEEE Power Enginecring Society Summer Meeting, 2000, vol. 1, pp. 463-468 vol. 1, 2000. 